\title{
Author Correction: Resource-diversity relationships in bacterial communities reflect the network structure of microbial metabolism
}

Martina Dal Bello (D), Hyunseok Lee (iD, Akshit Goyal and Jeff Gore (i)

Correction to: Nature Ecology \& Evolution https://doi.org/10.1038/s41559-021-01535-8, published online 19 August 2021

In the version of this Article initially published, Supplementary Table 1 was mistakenly omitted from the online Supplementary Information file. The table has now been included in the Supplementary Information file.

Published online: 15 September 2021

https://doi.org/10.1038/s41559-021-01563-4

(c) The Author(s), under exclusive licence to Springer Nature Limited 2021 